\title{
Improved Damping Constant of Hertz-Damp Model for Pounding between Structures
}

\author{
Dahai Zhao and Yong Liu \\ School of Civil Engineering and Mechanics, Yanshan University, No. 438 Hebei Road, \\ Qinhuangdao, Hebei, China \\ Correspondence should be addressed to Yong Liu; liuyongys11@163.com
}

Received 7 May 2016; Revised 10 July 2016; Accepted 17 July 2016

Academic Editor: Stefan Balint

Copyright (C) 2016 D. Zhao and Y. Liu. This is an open access article distributed under the Creative Commons Attribution License, which permits unrestricted use, distribution, and reproduction in any medium, provided the original work is properly cited.

Earthquake-induced pounding between structures has been widely studied by using different impact models. In this paper, we first briefly introduced the existing impact analysis models and discussed their shortcomings. For the Hertz-damp impact analysis model, the damping constants do not accord with the physical facts; then, based on the Hertz theory, the expression of the damping constant in the Hertz-damp impact analysis model is improved. The approximate formula of the damping constant is theoretically derived, and the accuracy and effectiveness of the calculation formula of the damping constant are verified by the selected collision analysis problems.

\section{Introduction}

Structural pounding is a complicated nonlinear problem. In order to effectively study the response of the adjacent structures under the impact, the reasonable impact analysis model is very significant. Quite a lot of scholars make a series of studies on the impact models. Since the Kelvin model is frequently used to simulate the collision of adjacent structures, the expression of the damping coefficient in Kelvin model has been derived by Ye et al. [1]. In order to preferably simulate the force and displacement relationship in the process of simulation, many scholars have used the Hertz theory to study the impact response. Jankowski [2] puts forward a nonlinear viscoelastic model based on the Hertz impact model and then derived the expression of the damping constant of the nonlinear viscoelastic impact model. The damping constant is related to the recovery coefficient, and the analytical formulation is verified by numerical simulations. Because the Hertz model does not consider the energy loss in the process of collision, Muthukumar and DesRoches [3] introduced the Hertz-damp model to be used in the impact analysis of structural engineering, which is compared with other impact models. And a lot of scholars $[4,5]$ have conducted in-depth research on the structural collision.
In this paper, based on the review of the main impact models and the Hertz theory, a new approximate formula of the damping constant of Hertz-damp model is derived. Meanwhile, the numerical simulation of the pounding analysis problems is conducted by MATLAB/Simulink software, the results of impact simulation show the correctness, accuracy, and effectiveness of the approximate formula of the damping constant of Hertz-damp model.

\section{Review of Existing Impact Models}

At present, the classical dynamic method and the contact element method are two main methods for the study of structural impact. As the classical dynamics method for the numerical analysis of the impact is not very convenient, generally rarely used, the second method is through establishment of the mathematical model of impact force for structure impact analysis, adding contact element at the impact site, which uses equivalent spring element and equivalent damper elements to, respectively, simulate interaction and energy dissipation during structural collision. Therefore, it is widely used. The following will be a brief review about the existing collision analysis models. 
2.1. Linear Elastic Model. The linear elastic model is the simplest kind of collision analysis model; the mathematical expression of the impact force is

$$
F_{c}= \begin{cases}k_{l} \delta & \delta>0 \\ 0 & \delta \leq 0,\end{cases}
$$

where $k_{l}$ is the impact spring stiffness and $\delta$ stands for the relative displacement of two colliding objects. This model does not consider the energy loss in the process of pounding, and the impact force is proportional to relative displacement $\delta$.

2.2. Kelvin Model. The Kelvin model is most widely used, and the linear viscous damping of Kelvin model is to represent the loss of energy. The impact force is expressed as

$$
F_{c}= \begin{cases}k_{k} \delta+c_{k} \dot{\delta} & \delta>0 \\ 0 & \delta \leq 0,\end{cases}
$$

where $\delta$ is the relative displacement of two colliding objects, $\dot{\delta}$ expresses the relative deformation velocity of the colliding objects during the impact process, $k_{k}$ is the impact spring stiffness of Kelvin model, and $c_{k}$ stands for the damping coefficient, which is determined by the following formula:

$$
c_{k}=2 \xi \sqrt{k_{k} \frac{m_{1} m_{2}}{m_{1}+m_{2}}},
$$

where $m_{1}$ and $m_{2}$ are the qualities of the colliding objects and $\xi$ stands for the damping constant, which is related to recovery coefficient $e$, and its expression is given by the following formula:

$$
\xi=-\frac{\ln e}{\sqrt{\pi^{2}+(\ln e)^{2}}} .
$$

The shortcoming of linear elastic impact models is that the viscous damping coefficient is kept constant during the whole collision process, which leads to a uniform change in energy loss at the impact start and rebound process; meanwhile, the Kelvin model will cause the impact force to show the pulling force at the impact beginning stage and emerge as leap at the impact rebound stage, and these phenomena are not consistent with the actual impact.

2.3. Hertz Model. In the impact process, the increase of the impact force simulated by Hertz model is nonlinear. In order to simulate the impact process more accurately, many scholars have studied the Hertz theory. The impact force based on Hertz theory is expressed as

$$
F_{c}= \begin{cases}k_{h} \delta^{3 / 2} & \delta>0 \\ 0 & \delta \leq 0,\end{cases}
$$

where $\delta$ stands for the relative displacement of two colliding objects and $k_{h}$ is the impact spring stiffness of Hertz model. However, Hertz model does not consider the energy loss in the impact process.
2.4. Hertz-Damp Model. The Hertz-damp model makes up the deficiency of the Hertz model without considering the energy loss and adds the nonlinear damping term. The relationship between the impact relative invasion displacement and the impact force is expressed as

$$
F_{c}= \begin{cases}k_{h} \delta^{3 / 2}+c_{h} \dot{\delta} & \delta>0 \\ 0 & \delta \leq 0,\end{cases}
$$

where $\delta$ is the relative displacement of two colliding objects, $\dot{\delta}$ expresses the relative deformation velocity of the colliding objects during the impact process, $k_{h}$ is the Hertz-damp model impact spring stiffness, and $c_{h}$ stands for the damping coefficient, and its expression is determined by the following formula:

$$
c_{h}=\xi \delta^{3 / 2}
$$

The relationship between damping constant $\xi$, nonlinear stiffness $k_{h}$, coefficient of restitution $e$, and the relative initial velocity of structure impact $\delta$ is expressed as

$$
\xi=\frac{3 k_{h}\left(1-e^{2}\right)}{4 \dot{\delta}} .
$$

In the nonlinear viscoelastic model, $\xi=0$ indicates that the impact is completely elastic impact and $\xi=\infty$ indicates that the impact is completely plastic impact [6]. Through (8), it can be found that $e=1$ and $\xi=0$ fits perfectly elastic impact; however $e=0$ and $\xi \neq \infty$ does not conform to the full plastic impact. Therefore, there are errors in the pounding simulation results simulated by the Hertz-damp model.

2.5. Nonlinear Viscoelastic Model. In the nonlinear viscoelastic model, Jankowski divided the collision process into the approach period and the restitution period. He thought that the cracking, crushing, and plastic deformation in the process of collision mainly occurred in the approach period; therefore, the energy loss mainly occurred in this stage. According to this, the Hertz-damp model was improved by Jankowski [6]. In the nonlinear viscoelastic model, the formula for the pounding force is expressed as follows:

$$
F_{c}= \begin{cases}k_{h} \delta^{3 / 2}+c_{h} \dot{\delta} & \dot{\delta}>0 \\ k_{h} \delta^{3 / 2} & \dot{\delta} \leq 0,\end{cases}
$$

where $\delta$ stands for the relative displacement of two colliding objects, $\dot{\delta}$ is the relative deformation velocity of the colliding objects during the impact process, $k_{h}$ expresses the impact spring stiffness of nonlinear viscoelastic model, and $c_{h}$ is the damping coefficient, its expression is determined by the following formula:

$$
c_{h}=2 \xi \sqrt{k_{h} \sqrt{\delta} \frac{m_{1} m_{2}}{m_{1}+m_{2}}} .
$$




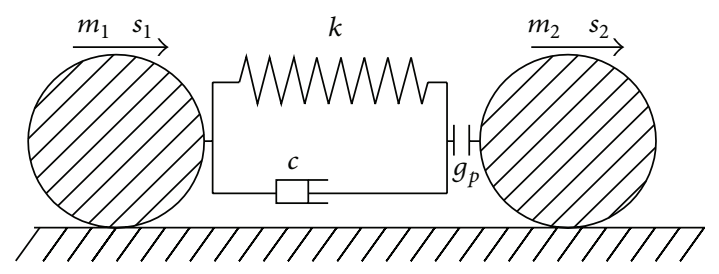

FIgURE 1: Analysis model of structural pounding.

The relationship between damping constant $\xi$ and coefficient of restitution $e$ is expressed as

$$
\xi=\frac{9 \sqrt{5}\left(1-e^{2}\right)}{2 e(e(9 \pi-16)+16)} .
$$

However, for the nonlinear viscoelastic model, the time history curve of the impact force simulated by the nonlinear viscoelastic model does not vary smoothly between the approach phase and restitution period of the collision [1], which is because there is a defect hypothesis in the process of velocity approximation formula derivation. All speed changes are added to the approach phase, which influences the coherence between the approach phase and restitution period of the collision. Therefore, the damping constant needs further optimization.

\section{Correction of Damping Constant}

In analysis model of structural pounding as shown in Figure $1, m_{1}$ and $m_{2}$ are the qualities of two colliding spheres; $s_{1}$ and $s_{2}$ stand for the displacements of two colliding spheres; $v_{1}$ and $v_{2}$ express respective velocities of two colliding spheres at the initial moment of contact; $v_{1}^{\prime}$ and $v_{2}^{\prime}$ are the velocities of colliding spheres after impact; $c$ stands for the damping coefficient; $g_{p}$ is the initial clearance of the colliding spheres; $k$ expresses the stiffness coefficient of impact contact element.

According to classical mechanics, energy loss $\Delta E$ caused by the collision can be expressed as a function of coefficient of restitution $e$ and the relative velocity of two colliding objects [7]:

$$
\Delta E=\frac{1}{2} \frac{m_{1} m_{2}}{m_{1}+m_{2}}\left(1-e^{2}\right)\left(v_{1}-v_{2}\right)^{2} .
$$

According to the classical theory of impact, coefficient of restitution $e$ can be calculated from the following equation:

$$
e=\frac{v_{2}^{\prime}-v_{1}^{\prime}}{v_{1}-v_{2}}
$$

At the same time, the energy loss dissipated by the damper can be expressed as

$$
\Delta E=\int_{0}^{\delta_{m}} c_{h} \dot{\delta} d \delta
$$

The variation of interpenetration displacement $\delta$ during impact is illustrated in Figure 2, where $t^{-}, t^{m}$, and $t^{+}$stand

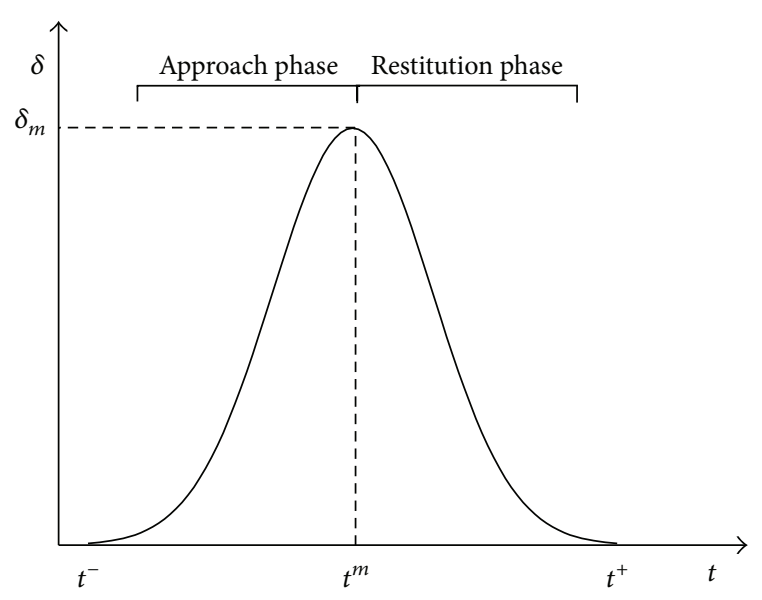

FIGURE 2: Typical time variation of interpenetration during impact.

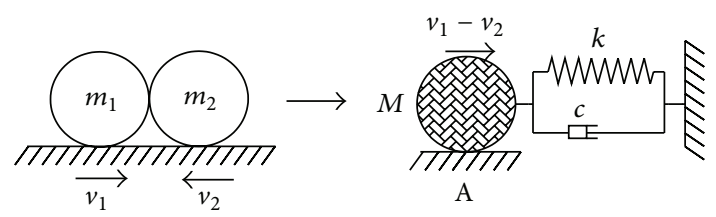

FIGURE 3: Equivalent model of impact between two colliding bodies.

for the initial time of contact, the time which maximum interpenetration displacement $\delta_{m}$ occurs and the time of separation between the two colliding bodies.

The two degrees of freedom system shown in Figure 3 can be equivalent to a single degree of freedom system, which can be seen as a single mass, spring, and damper model, as shown in Figure 3. The definition of equivalent model is as follows: (1) the spring stiffness and damping are the same as that of two degrees of freedom system; (2) the mass of the equivalent model is equivalent masses of the two colliding bodies: $M=m_{1} m_{2} /\left(m_{1}+m_{2}\right)$; (3) in Figure 3, A represents the initial point and the initial interpenetration displacement is $\delta_{0}=0$, and the initial interpenetration velocity is $\dot{\delta}_{0}=v_{1}-v_{2}$, when the maximum displacement point is reached, $\delta=\delta_{m}, \dot{\delta}=0$, among them, $\delta_{m}$ corresponds to the maximum interpenetration displacement in the collision process. The approach phase is represented from A to the maximum displacement point, the same way from the maximum displacement point to $\mathrm{A}$ indicates the restitution phase. In this single degree of freedom system, the equation of motion of the equivalent model is derived as

$$
M \ddot{\delta}+c_{h} \dot{\delta}+k_{h} \delta^{n}=0
$$

where $M=m_{1} m_{2} /\left(m_{1}+m_{2}\right)$ stands for the equivalent mass of the single degree of freedom system, when $n=1.5$, it represents Hertz-damp model. When $n \neq 1$, the analytical solution of the equation of motion (15) will be very difficult to be found; however, it can be solved by a simple iterative method. 
Using $p$ to replace $\dot{\delta}[8]$, then

$$
\ddot{\delta}=p \frac{d p}{d \delta} .
$$

Substituting (16) into (15), we can get

$$
M p \frac{d p}{d \delta}+c_{h} p+k_{h} \delta^{3 / 2}=0 .
$$

Employing the traditional separation of variables, ignoring the influence of the damping based on the Hertz contact theory, and integrating (17) yield

$$
M \int_{\dot{\delta}_{f}}^{\dot{\delta}} p d p=-k_{h} \int_{0}^{\delta} \delta^{3 / 2} d \delta
$$

where $\dot{\delta}$ is the interpenetration velocity, $\dot{\delta}_{f}$ stands for the interpenetration velocity at the moment of collision separation, and $\delta$ expresses the interpenetration displacement.

After integral calculation, one obtains

$$
\frac{1}{2} M\left(\dot{\delta}^{2}-\dot{\delta}_{f}^{2}\right)=-\frac{2}{5} k_{h} \delta^{2 / 5} .
$$

Transformation form of (19) and then the displacement deformation $\delta$ can be expressed as

$$
\delta=\left[\frac{5 M}{4 k_{h}}\left(\dot{\delta}_{f}^{2}-\dot{\delta}^{2}\right)\right]^{2 / 5} .
$$

Maximum interpenetration displacement $\delta_{m}$ can be expressed as

$$
\delta_{m}=\left[\frac{5 M}{4 k_{h}} \dot{\delta}_{f}^{2}\right]^{2 / 5} .
$$

Based upon (20) and (21), the relationship between $\delta$ and $\dot{\delta}$ can be get:

$$
\left(\frac{\delta}{\delta_{m}}\right)^{5 / 2}+\left(\frac{\dot{\delta}}{\dot{\delta}_{f}}\right)=1
$$

From (22), the interpenetration velocity $\dot{\delta}$ at the restitution period can be obtained:

$$
\dot{\delta}=-\dot{\delta}_{f} \sqrt{1-\left(\frac{\delta}{\delta_{m}}\right)^{5 / 2}},
$$

where $\dot{\delta}_{f}=v_{1}^{\prime}-v_{2}^{\prime}$ indicates the interpenetration velocity of two objects at the moment of separation.

Substituting (23) into (14), the energy loss $\Delta E$ caused by the damping force can be expressed as

$$
\Delta E=-2 \xi \sqrt{k_{h} M} \int_{0}^{\delta_{m}} \delta^{1 / 4} \dot{\delta}_{f} \sqrt{1-\left(\frac{\delta}{\delta_{m}}\right)^{5 / 2}} d \delta .
$$

The evaluation of the integral of (24) can be found elsewhere [9]. Applying this method to solve the following equation,

$$
\Delta E=-2 \xi \dot{\delta}_{f} \sqrt{k_{h} M} \delta_{m}^{5 / 4} .
$$

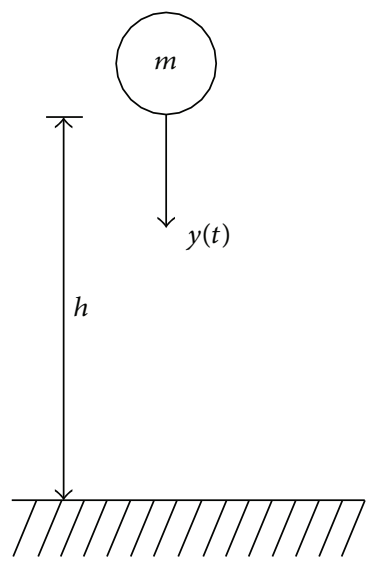

FiguRE 4: Numerical model of a ball falling on a rigid surface.

Equating (25) and (12) leads to

$$
\frac{1}{2} \frac{m_{1} m_{2}}{m_{1}+m_{2}}\left(1-e^{2}\right)\left(v_{1}-v_{2}\right)^{2}=-2 \xi \dot{\delta}_{f} \sqrt{k_{h} M} \delta_{m}^{5 / 4} .
$$

Substituting (21) into (26), one obtains

$$
\frac{1}{2} M\left(1-e^{2}\right)\left(v_{1}-v_{2}\right)^{2}=-\sqrt{5} M \xi \dot{\delta}_{f}\left|\dot{\delta}_{f}\right| .
$$

Then, the expression of improved damping constant $\xi$ can be obtained:

$$
\xi=\frac{\sqrt{5}\left(1-e^{2}\right)}{10 e^{2}} .
$$

\section{Numerical Verification}

In order to verify the correctness of the theoretical derivation for the expression of damping constant $\xi$, the following two selected collision analysis problems are selected for numerical verification. The procedures for the verification are as follows: first, selecting the collision analysis problem and determining the initial value of the coefficient of restitution $e_{0}$, which varies from 0.1 to 1.0 with interval 0.1 ; then, obtaining the numerical solution of the coefficient of restitution $e$ by the collision analysis based on the impact models; and finally comparing relative error [5] $\left|e_{0}-e\right| / e_{0}$ between the initial value of the coefficient of restitution $e_{0}$ and the numerical solution of the coefficient of restitution $e$.

4.1. The Collision between Sphere and Static Rigid Plane. The collision between the steel ball and the rigid surface by the free-falling [2] is shown in Figure 4. The following parameters have been used: $m=0.5 \mathrm{~kg}, h=0.5 \mathrm{~m}$, and $k_{h}=1 \times$ $10^{10} \mathrm{~N} / \mathrm{m}^{3 / 2}$. The dynamic equation of motion for such a model can be obtained by analyzing the form as follows:

$$
m \ddot{y}(t)+F_{c}(t)=m g,
$$

where $m$ is the quality of falling ball, $\ddot{y}(t)$ stands for the vertical acceleration of falling ball, $y(t)$ expresses its vertical 


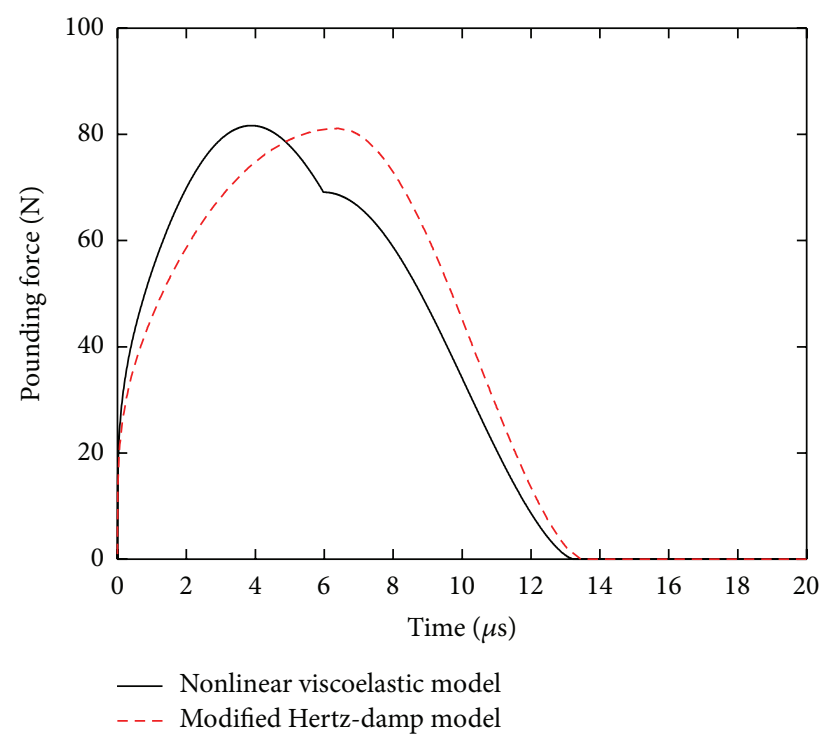

Figure 5: Pounding force time histories during impact between falling steel ball and static rigid plane.

displacement, $g$ indicates the acceleration of gravity, $h$ is the drop height of falling ball, impact force $F_{c}(t)$ is calculated according to (6) and (9), and impact deformation $\delta$ is counted as

$$
\delta(t)=y(t)-h .
$$

The previously described nonlinear viscoelastic impact model together with the Hertz-damp model with the improved damping constant is used to simulate this collision analysis problem, which has been conducted for impacts analysis of a ball falling on a rigid surface applying various impact models [2]. In this paper, a set of data, the maximum impact force of $80.7 \mathrm{~N}$ in the test, is selected for numerical simulation, and the appropriate values of impact parameters $\left(k_{h}=1 \times 10^{10} \mathrm{~N} / \mathrm{m}^{3 / 2}, e=0.65\right)$ have been obtained numerically through iterative simulations in order to satisfy the maximum impact force. A time-stepping integration procedure written through MATLAB/Simulink software with the fixed time increment step $\Delta t=1 \times 10^{-7} \mathrm{~s}$ has been applied to solve (29), the impact force time history curves of the nonlinear viscoelastic impact and the Hertz-damp model with the improved damping constant are presented in Figure 5.

4.2. The Collision between the Pendulum and the Static Rigid Wall. In order to further verify the correctness of the approximate formula of damping constant $\xi$, the collision between the pendulum and the static rigid wall [10] is as shown in Figure 6. The following parameters have been used: $m=570 \mathrm{~kg}, L=0.7 \mathrm{~m}$, and $k_{h}=2 \times 10^{9} \mathrm{~N} / \mathrm{m}^{3 / 2}$ and the initial impact velocity is $v_{0}=0.5 \mathrm{~m} / \mathrm{s}$. The motion dynamic equation of this model can be expressed as follows:

$$
m \ddot{x}(t)+\frac{m g}{L} x(t)+F_{c}(t)=0,
$$

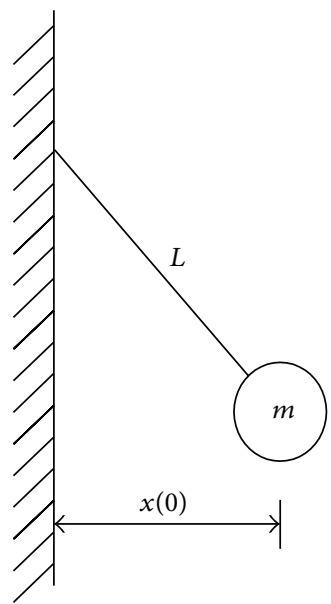

FIGURE 6: Model of a pendulum ball which strikes a rigid barrier.

where $m$ is the mass of the pendulum striker, $\ddot{x}(t)$ and $x(t)$, respectively, express the horizontal acceleration and horizontal displacement of the pendulum striker, $L$ stands for the length of the pendulum striker, $g$ expresses the acceleration of gravity, and $F_{c}(t)$ stands for the impact force, which is calculated, respectively, according to (6) and (9), in which the deformation $\delta(t)$ is expressed as

$$
\delta(t)=x(t) .
$$

The numerical analysis has been conducted for impacts of the pendulum and the static rigid wall applying the nonlinear viscoelastic impact and the Hertz-damp model with the improved damping constant. In this paper, the maximum impact force of $102.5 \mathrm{kN}$ in the test [2] is selected for numerical analysis, and the appropriate values of impact parameters $\left(k_{h}=2 \times 10^{9} \mathrm{~N} / \mathrm{m}^{3 / 2}, e=0.65\right)$ have been determined through iterative procedures in order to attain the maximum impact force. A time-stepping integration procedure written by MATLAB/Simulink software with constant time step $\Delta t=$ $1 \times 10^{-4} \mathrm{~s}$ has been used for solving (31) numerically and the time history curves of the impact force of different impact models are shown in Figure 7.

4.3. Comparison between Analytical Formulations and Numerical Results. The analytical formulation, which is related to the impact damping ratio $\xi$ and the coefficient of restitution $e$, has been derived as shown in (28), the comparison with the numerical results has been carried out as shown in Figure 8 for verifying the accuracy and effectiveness of the expression. As shown above, the numerical studies have been conducted for pounding with nonlinear viscoelastic model and modified Hertz-damp model.

Simulation results of different impact models and the relative error percentages of recovery coefficient at different initial values are presented in Tables 1 and 2. As can be seen from the tables, the impact model of this paper has higher simulation precision than the nonlinear viscoelastic model and Hertz-damp model. At the same time, the relative error 
TABLE 1: Relative error of recovery coefficient under the collision between sphere and static rigid plane.

\begin{tabular}{|c|c|c|c|c|c|c|}
\hline \multirow{2}{*}{$e_{0}$} & \multicolumn{2}{|c|}{ The model of this paper } & \multicolumn{2}{|c|}{ Nonlinear viscoelastic model } & \multicolumn{2}{|c|}{ Hertz-damp model } \\
\hline & $e$ & Error $(\%)$ & $e$ & Error (\%) & $e$ & Error (\%) \\
\hline 0.1 & 0.0724 & 27.6 & 0.0680 & 32.0 & 0.6639 & 563.9 \\
\hline 0.2 & 0.1618 & 19.1 & 0.1544 & 22.8 & 0.6715 & 235.8 \\
\hline 0.3 & 0.2690 & 10.3 & 0.2568 & 14.4 & 0.6841 & 128.0 \\
\hline 0.4 & 0.3779 & 5.5 & 0.3619 & 9.5 & 0.7024 & 75.6 \\
\hline 0.5 & 0.4831 & 3.4 & 0.4710 & 5.8 & 0.7258 & 45.2 \\
\hline 0.6 & 0.5916 & 1.4 & 0.5762 & 4.0 & 0.7563 & 26.1 \\
\hline 0.7 & 0.6937 & 0.9 & 0.6827 & 2.5 & 0.7960 & 13.7 \\
\hline 0.8 & 0.7965 & 0.4 & 0.7874 & 1.6 & 0.8477 & 6.0 \\
\hline 0.9 & 0.9000 & 0.0 & 0.8936 & 0.7 & 0.9142 & 1.6 \\
\hline 1.0 & 1.0000 & 0.0 & 1.0000 & 0.0 & 1.0000 & 0.0 \\
\hline
\end{tabular}

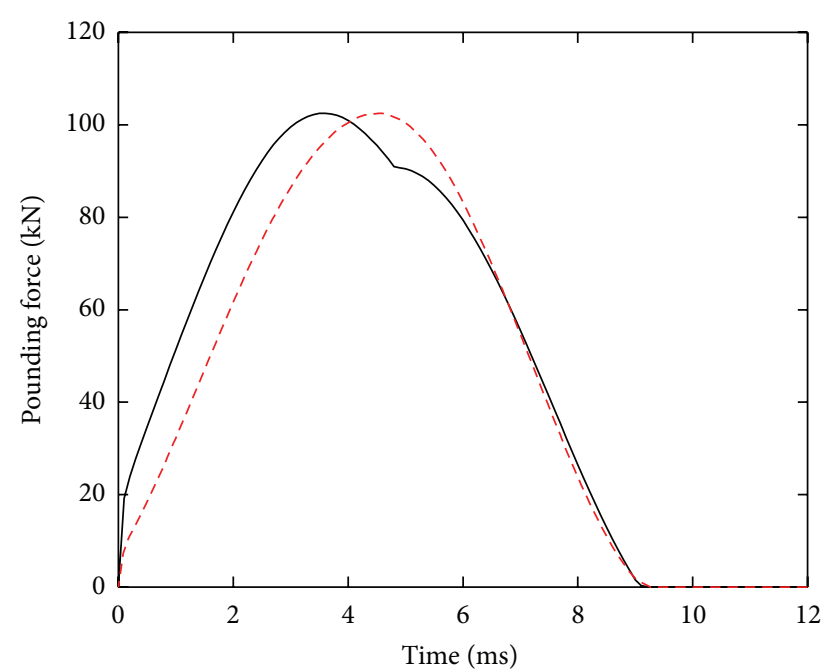

- Nonlinear viscoelastic model

- - - Modified Hertz-damp model

FIGURE 7: Pounding force time histories during impact between the pendulum and the static rigid wall.

decreases with the increase of initial value $e_{0}$, and when the initial value of the coefficient of restitution is greater than 0.5 , the relative error is less than $1.5 \%$.

Contrasting pounding force histories presented in Figures 5 and 7, it can be seen that the time history curves of the impact force simulated by the modified Hertz-damp model vary smoothly; however, impact force curves simulated by the nonlinear viscoelastic model do not vary consistently at a certain moment, which is the same as the simulation results by Jankowski [2]; at the same time, the simulation results obtained by modified Hertz-damp are closer to actual value than that simulated by nonlinear viscoelastic model at approach phase, and the simulation results by these two models are relatively close during the restitution period.

Through Figure 8, it can be seen that two analytical formulations can be used to express well the relationship between the impact damping ratio $\xi$ and the coefficient of restitution $e$; however, by carefully observing, we can find

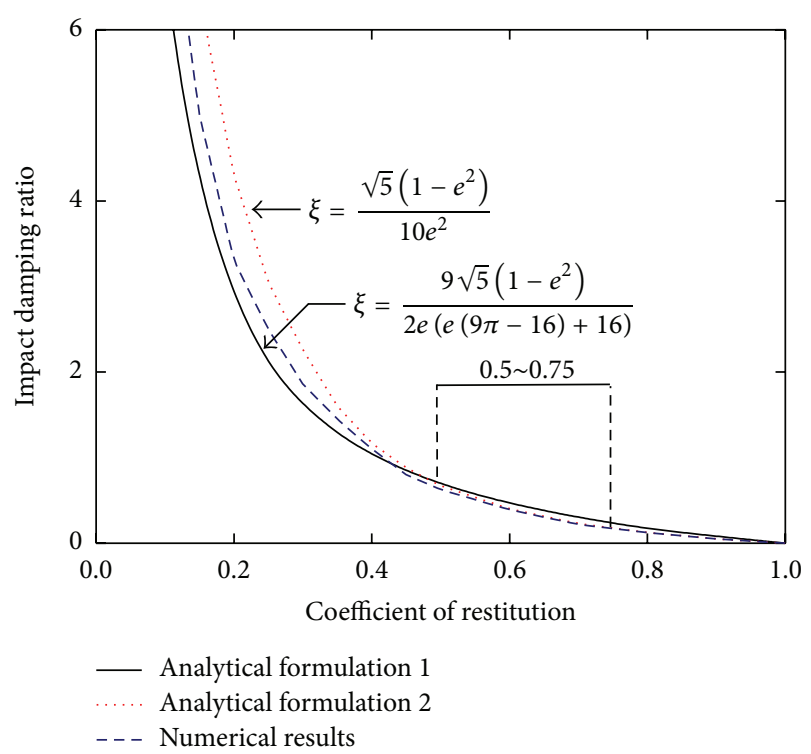

FIGURE 8: Comparison between analytical formulations and numerical results concerning the relation between the impact damping ratio and the coefficient of restitution.

that analytical formulation 2 can quite accurately simulate the relation between the impact damping ratio $\xi$ and the coefficient of restitution $e$ between 0.5 and 0.75 . As we all know, in the collision of the actual engineering structure, it has been assessed that the coefficient of restitution $e$ is generally used to simulate real collisions between structures and ranges usually from 0.5 to 0.75 [7]; thus it can be concluded that reliable results of impact simulation in structural engineering can be provided by using the improved damping constant formula.

\section{Conclusions}

This paper first reviews the existing structure impact analysis models and discusses the problems between them; then, the damping constant $\xi$ of the Hertz-damp model is theoretically rededuced according to Hertz theory, and the accuracy and effectiveness of the damping constant are verified by numerical simulation. In the practical structure engineering, 
TABLE 2: Relative error of recovery coefficient under the collision between the pendulum and the static rigid wall.

\begin{tabular}{|c|c|c|c|c|c|c|}
\hline \multirow{2}{*}{$e_{0}$} & \multicolumn{2}{|c|}{ The model of this paper } & \multicolumn{2}{|c|}{ Nonlinear viscoelastic model } & \multicolumn{2}{|c|}{ Hertz-damp model } \\
\hline & $e$ & Error (\%) & $e$ & Error (\%) & $e$ & Error (\%) \\
\hline 0.1 & 0.0738 & 26.2 & 0.0688 & 31.2 & 0.6604 & 560.4 \\
\hline 0.2 & 0.1644 & 17.8 & 0.1550 & 22.5 & 0.6698 & 234.9 \\
\hline 0.3 & 0.2696 & 10.1 & 0.2557 & 14.8 & 0.6835 & 127.8 \\
\hline 0.4 & 0.3785 & 5.3 & 0.3622 & 9.5 & 0.7015 & 75.4 \\
\hline 0.5 & 0.4857 & 2.9 & 0.4692 & 6.2 & 0.7250 & 45.0 \\
\hline 0.6 & 0.5921 & 1.3 & 0.5769 & 3.9 & 0.7553 & 25.9 \\
\hline 0.7 & 0.6949 & 0.7 & 0.6826 & 2.5 & 0.7955 & 13.6 \\
\hline 0.8 & 0.7976 & 0.3 & 0.7869 & 1.6 & 0.8481 & 6.0 \\
\hline 0.9 & 0.9000 & 0.0 & 0.8948 & 0.6 & 0.9137 & 1.5 \\
\hline 1.0 & 1.0000 & 0.0 & 1.0000 & 0.0 & 1.0000 & 0.0 \\
\hline
\end{tabular}

more reliable results can be obtained by using the Hertzdamp model with the improved damping constant.

\section{Competing Interests}

The authors declare no competing interests regarding the publication of this paper.

\section{Acknowledgments}

This research was supported by National Natural Science Foundation of China under Grant no. 51308487, no. 41402261, and no. 51408526, Heibei Provincial Natural Science Foundation of China under Grant no. E2014203055, and the Excellent Youth Foundation of Hebei Educational Committee under Grant no. YQ2013015. The support for this research is greatly appreciated.

\section{References}

[1] K. Ye, L. Li, and H. Zhu, "A modified Kelvin impact model for pounding simulation of base-isolated building with adjacent structures," Earthquake Engineering and Engineering Vibration, vol. 8, no. 3, pp. 433-446, 2009.

[2] R. Jankowski, "Non-linear viscoelastic modelling of earthquake-induced structural pounding," Earthquake Engineering and Structural Dynamics, vol. 34, no. 6, pp. 595-611, 2005.

[3] S. Muthukumar and R. DesRoches, "A Hertz contact model with non-linear damping for pounding simulation," Earthquake Engineering \& Structural Dynamics, vol. 35, no. 7, pp. 811-828, 2006.

[4] Y. Liu, W.-G. Liu, X. Wang, W.-F. He, and Q.-R. Yang, "New equivalent linear impact model for simulation of seismic isolated structure pounding against moat wall," Shock and Vibration, vol. 2014, Article ID 151237, 10 pages, 2014.

[5] M. Ismail, "Inner pounding control of the RNC isolator and its impact on seismic isolation efficiency under near-fault earthquakes," Engineering Structures, vol. 86, pp. 99-121, 2015.

[6] R. Jankowski, "Analytical expression between the impact damping ratio and the coefficient of restitution in the non-linear viscoelastic model of structural pounding," Earthquake Engineering \& Structural Dynamics, vol. 35, no. 4, pp. 517-524, 2006.
[7] K. Ye, L. Li, and H. Zhu, "A note on the Hertz contact model with nonlinear damping for pounding simulation," Earthquake Engineering and Structural Dynamics, vol. 38, no. 9, pp. 1135$1142,2009$.

[8] P. Liu, K.-F. Zheng, W. He, and H. Liu, "Improved algorithm of contact element model for earthquake-induced pounding between structures," Journal of Chongqing University, vol. 35, no. 2, pp. 135-142, 2012.

[9] C. H. Edwards and D. E. Penney, Calculus and Analytic Geometry, Prentice Hall, Englewood Cliffs, NJ, USA, 1st edition, 1982.

[10] X. Xiang, L. Weiqing, and X. Xiuli, "Linear viscoelastic model of earthquake-induced structural pounding," Engineering Mechanics, vol. 30, no. 2, pp. 278-284, 2013. 


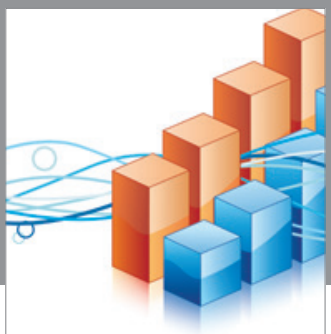

Advances in

Operations Research

vatem alat4

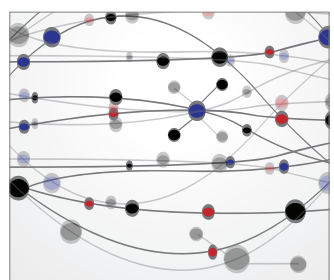

\section{The Scientific} World Journal
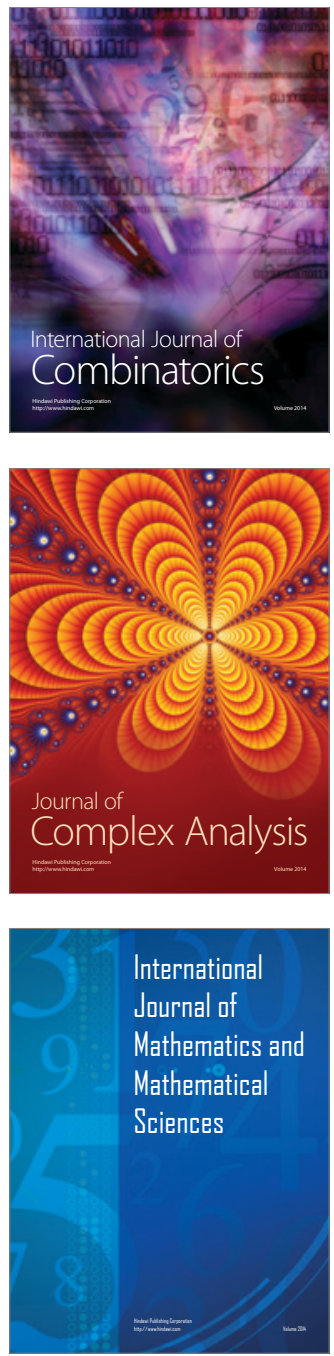
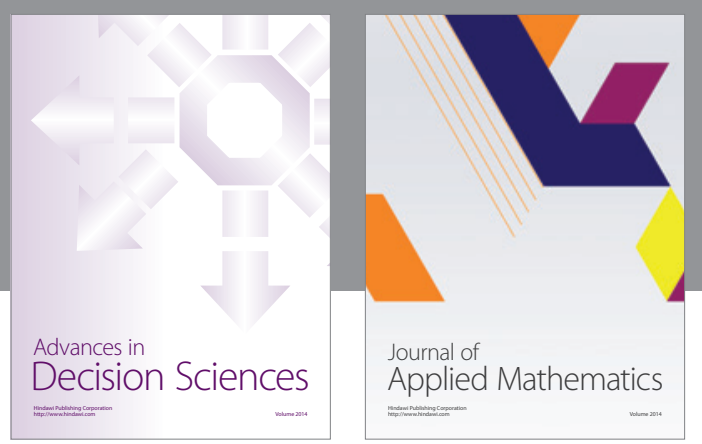

Algebra

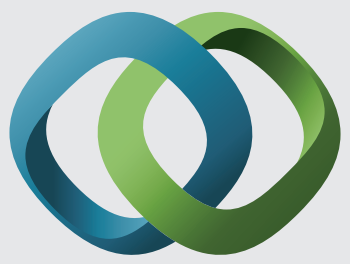

\section{Hindawi}

Submit your manuscripts at

http://www.hindawi.com
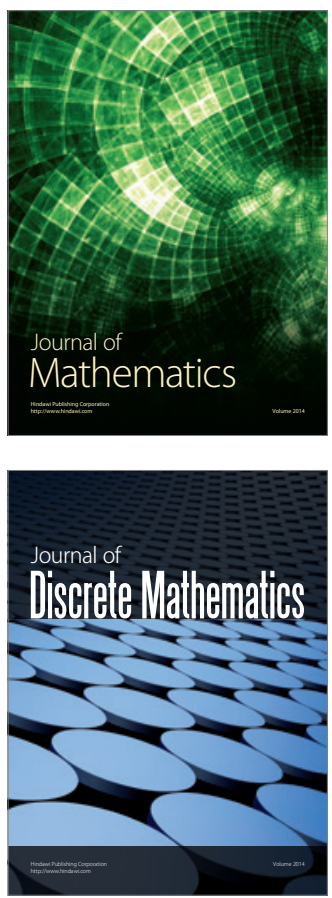

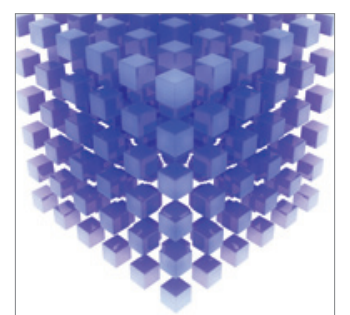

Mathematical Problems in Engineering
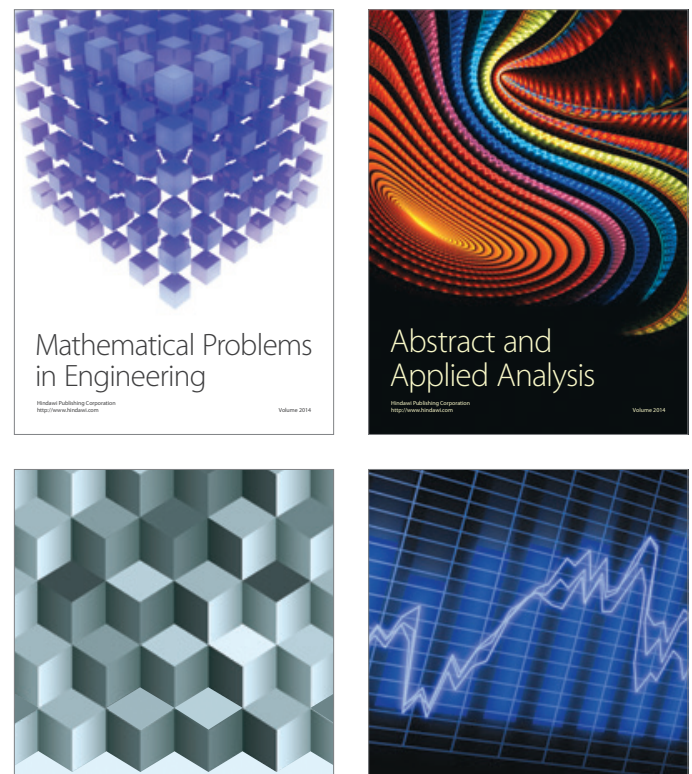

Journal of

Function Spaces

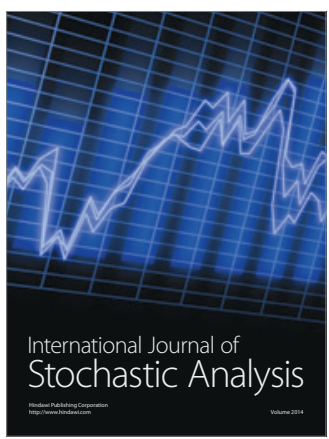

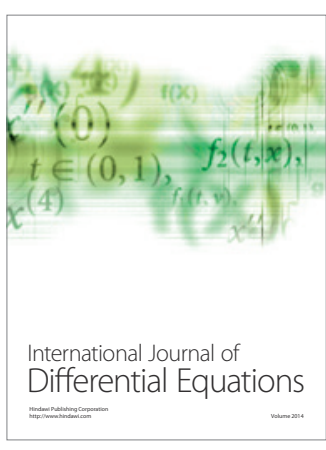
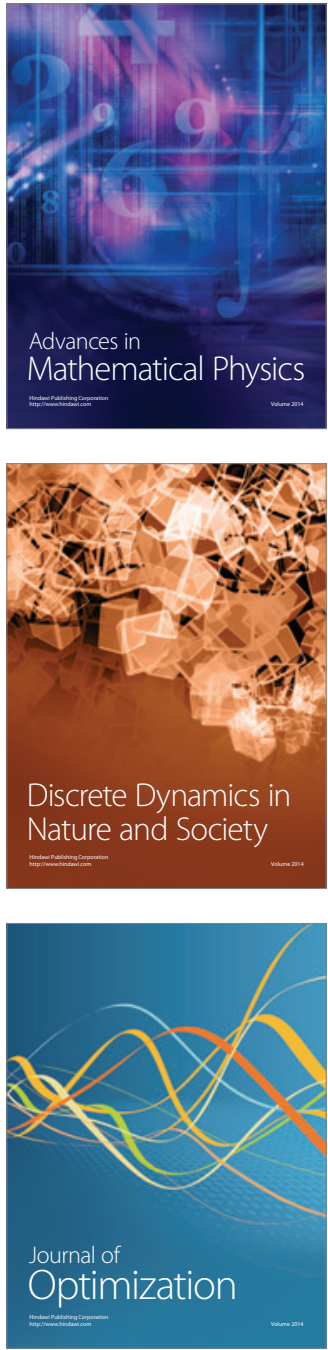